\title{
Performance Analysis and Node Selection of Intelligent Reflecting Surface-Aided Visible Light Communication for Parallel Vehicles
}

\author{
Ling Zhan $\mathbb{D}^{1,2}$ Hong Zhao $\mathbb{D}^{2},{ }^{2}$ Wenhui Zhang, ${ }^{3}$ Jiming Lin $\mathbb{D}^{4},{ }^{4}$ and Xiang Zhao $\mathbb{D}^{2}$ \\ ${ }^{1}$ Guangxi Key Laboratory of Wireless Wideband Communication and Signal Processing, Guilin University of Electronic Technology, \\ Guilin 541004, China \\ ${ }^{2}$ School of Information and Communication, Guilin University of Electronic Technology, Guilin 541004, China \\ ${ }^{3}$ School of Computer Science and Information Security, Guilin University of Electronic Technology, Guilin 541004, China \\ ${ }^{4}$ College of Electronic and Information Engineering, Beibu Gulf University, Qinzhou 535011, China
}

Correspondence should be addressed to Hong Zhao; zhaohong@guet.edu.cn and Jiming Lin; zhang_linjm@163.com

Received 26 November 2021; Revised 28 January 2022; Accepted 15 February 2022; Published 28 February 2022

Academic Editor: Li Zhu

Copyright (c) 2022 Ling Zhan et al. This is an open access article distributed under the Creative Commons Attribution License, which permits unrestricted use, distribution, and reproduction in any medium, provided the original work is properly cited.

In the visible light communication (VLC) for parallel vehicles, the light emitted by the headlamps cannot reach the photodiode (PD), which is the receiver installed on another vehicle. To solve this problem, the mirror array-based intelligent reflecting surface- (IRS-) aided VLC system is designed for parallel vehicles, and the system performance with different number of mirrors and transmission distance is analyzed. The maximum distance between adjacent IRSs is calculated via the exhaustive method, and the nearest neighbor iterative search (NNIS) algorithm is proposed for IRS node selection. Numerical results show that the SNR increases with the number of mirrors increasing. If the number of mirrors is $6 \times 6$ in the IRS, the maximum distance between adjacent IRSs is 37 meters. When the interval between adjacent IRSs is 32 meters, the required BER can be satisfied with merely three IRSs working together according to the NNIS algorithm.

\section{Introduction}

Due to the continuous increase in the number of vehicles resulting in worsening road congestion and increasing traffic accidents, the requirement for traffic management must be enhanced [1]. The intelligent transportation system (ITS) [2, 3 ] is a safe, convenient, and efficient traffic management system for promoting the coordination of various traffic elements and realizing information sharing [4]. It mainly includes inside and outside vehicle communication $[5,6]$. The latter includes the communication between the vehicle and its surrounding environment, such as vehicle-to-vehicle (V2V), vehicle-toinfrastructure (V2I), infrastructure-to-vehicle (I2V), vehicleto-cloud, vehicle-to-pedestrians, and vehicles-to-grid [7, 8]. IEEE $802.11 \mathrm{p}$ standard defines dedicated short-range communication (DSRC) which is one of the most promising wireless standards to support ITS $[9,10]$. However, the complex traffic environment, insufficient frequency band, various obstacles, and serious Doppler effect pose present considerable challenges to radiofrequency- (RF-) based ITS.

With its low price and green and energy-saving features, LED has gradually replaced traditional light sources in recent years [11]. The number of headlights, taillights, brake lights, and daytime running lights of vehicles using LED is increasing [12], providing hardware support for vehicular visible light communication (VVLC). In the V2V VLC system, headlights [13], taillights [14], or even brake lights [15] can be used as the transmitter, and the image sensor [16], high-speed camera [17], or PD [18] can be used as the receiver. VLC can be used as a supplement to RF communications [19] solving the problems such as insufficient frequency band, slow transmission speed, and Doppler effect.

Currently, the studies on V2V VLC mainly focus on the line-of-sight (LOS) links that the light can directly reach the receiver from the transmitter [20-22]. Although V2V VLC has achieved good results in the LOS link, all the light 
emitted by the transmitter cannot possibly be within the field of view (FOV) of the receiver [23]. If the vehicles are driving side by side in different lanes or the two vehicles have obstacles between them (such as other vehicles), the light from the transmitter is blocked, resulting in VLC interruption. VLC with the nonline-of-sight (NLOS) links must be studied to solve this problem. When the light from headlights of the back vehicle irradiates on the asphalt pavements [24] or the road surface with water [25], the reflected light can reach the receiver installed on the front vehicle, realizing the VLC through the NLOS links. However, the reflection coefficient of asphalt pavements is low. Although the reflection coefficient of the water surface is high, it is easy to shake. Thus, the reflecting surface that satisfies the conditions cannot be guaranteed to appear in real time.

The intelligent reflecting surface (IRS) $[26,27]$ is a tunable metasurface composed of many passive and low-cost passive reflective elements. This metasurface can realize the functions of traditional optical devices [28, 29]. For the metasurfacebased IRS model, beam steering [30], beam shaping [31], and improvement of link service level [32] of coherent light have been studied. For visible light, Abdelhady et al. [33] install the IRS on the wall to reflect the incident light to the receiver by intelligently controlling the phase gradient of each subsurface and the direction of each mirror which provide convenience in solving the communication interruption caused by blind zone in the V2V VLC system.

Given the continuous improvement of transportation infrastructures and the good performance of the mirror array-based IRS, we design the mirror array-based IRSaided VLC system for parallel vehicles to solve the VLC interruption problem, and the performances of different numbers of mirrors in the IRS are compared. Moreover, the influence of the distance between adjacent IRSs is analyzed, and the IRS node selection algorithm is proposed.

The contributions of this paper are as follows:

(1) The mirror array-based IRS-aided VLC system for parallel vehicles is designed for the first time that IRS can be installed on transportation infrastructure as the reflection point of the light. The light is reflected to the receiver through the intelligent controlling the rotation angles of the mirrors which provides a new approach for parallel vehicles to communicate via visible light

(2) The different numbers of mirrors in the IRS affect the received power. In this study, the system performance under the different numbers of mirrors in the IRS is analyzed to provide a basis for selecting the appropriate number of mirrors

(3) The distance between adjacent IRSs affects the direction of the reflected light and the received optical power. In this study, the influence of the distance between adjacent IRSs is analyzed, and the maximum distance between adjacent IRSs that can satisfy the communication requirements is calculated through an exhaustive method
(4) Multiple IRSs can be installed within the maximum distance between adjacent IRSs according to the actual distance between transportation infrastructures. The nearest neighbor iterative search (NNIS) algorithm is proposed for selecting IRS nodes. The least IRS nodes can be selected to satisfy the communication requirements, and the resource utilization of IRS is improved

The remainder of the paper is organized as follows. Section 2 introduces the mirror array-based IRS-aided VLC system for parallel vehicles, presents the expressions of the channel gain and SNR, and analyzes the influence of the number of mirrors and optical transmission distance. Section 3 introduces the system model of multiple IRS-aided V2V VLC, presents the calculation method of the maximum distance between adjacent IRSs, and proposes the NNIS algorithm for selecting IRS nodes. Section 4 provides the simulation parameters and the numerical results. The conclusions and future research directions are drawn in Section 5.

\section{The Mirror Array-Based IRS-Aided VLC for Parallel Vehicles}

For two parallel vehicles, the light from the transmitter may not be able to reach the receiver, resulting in the blind zone of VLC. At this time, the aided equipment should be considered to realize VLC. The mirror array-based IRS has been proved to be an effective approach in indoor VLC [33]. This approach is applied to the V2V VLC system in this study, and the performance is analyzed.

2.1. System Model. The application scenario of the mirror array-based IRS-aided VLC system for parallel vehicles is shown in Figure 1. Given that the vehicle is constantly moving, the direction of the reflected light must be changed with the distance between the vehicle and the IRS to enable that the light emitted by the headlamp reaches the receiver in any case. The mirror array-based IRS can satisfy this requirement. With the aid of the IRS, the light from the transmitter reaches the IRS first. Given the excellent reflection characteristics of the mirror, the light can be reflected to the receiver according to the communication requirements, thereby realizing VLC.

Two adjacent lanes are taken as an example to visually analyze the performance. The vehicles run along the central axis of the lanes, and the right headlamp of the vehicle in the right lane serves as the transmitter. The $\mathrm{PD}$ is installed in the middle of the two headlamps of the vehicle in the left lane as the receiver to avoid driver dizziness caused by reflected light. The IRS is installed on the street light pole, and the height of the center point is consistent with that of the headlamps. Figure 2 shows the model of the system.

For the mirror array-based IRS, the number of mirrors in each row and each column can be set as $n_{k}$ and $n_{l}$, respectively. The width of each mirror is $w_{m}$, and the height is $h_{m}$. $\Delta w_{m}$ and $\Delta h_{m}$ are the edge-to-edge intermirror separation distances along the $x$-axis and $z$-axis. We define a Cartesian coordinate system whose origin is at the center of the mirror $R_{i, j}\left(1 \leq i \leq n_{k}, 1 \leq j \leq n_{l}\right)$. We can obtain the position vector of the transmitter $\mathrm{S}$. 


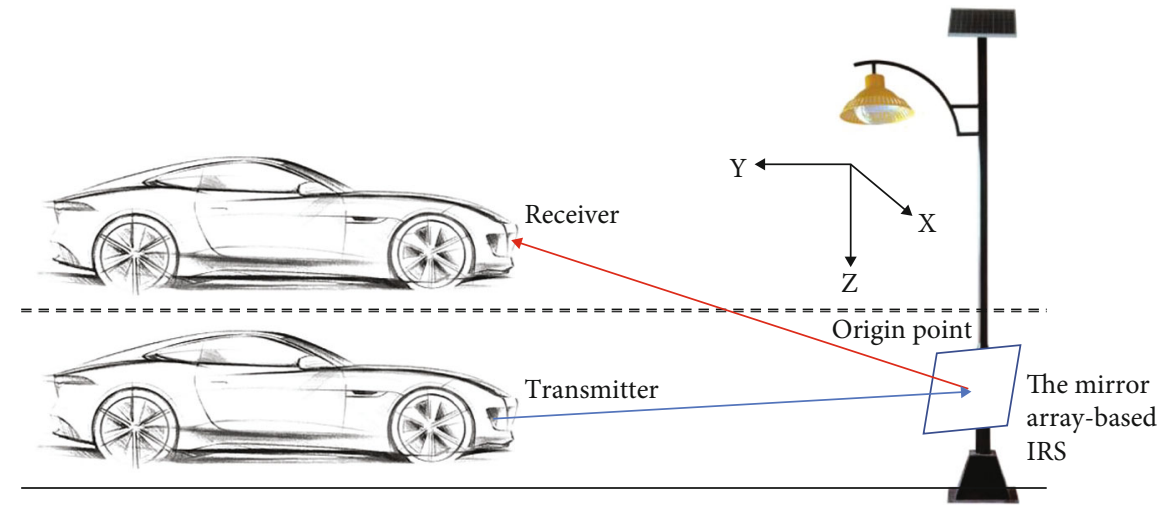

FIGURE 1: Application scenario of the mirror array-based IRS-aided VLC system for parallel vehicles.

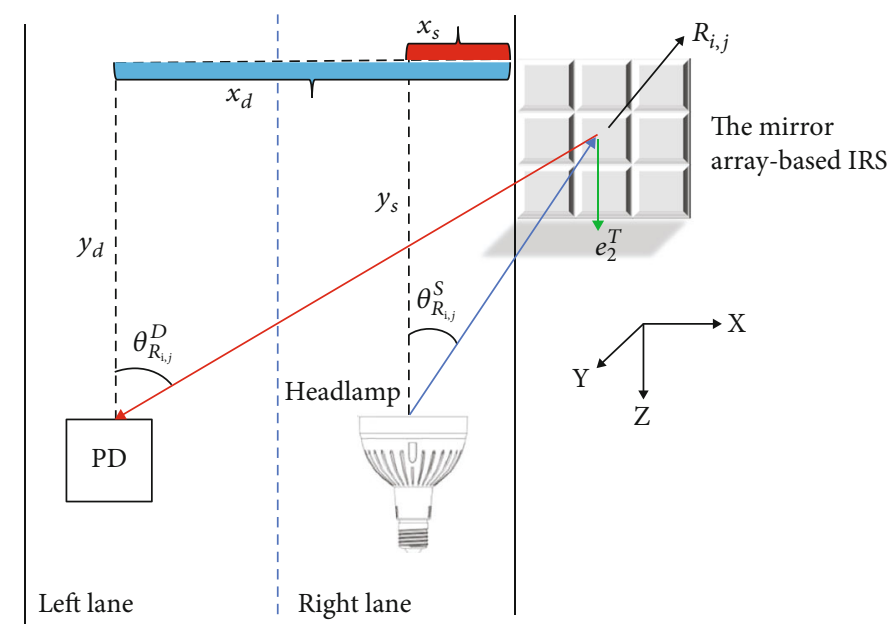

FIgURe 2: Model of the mirror array-based IRS-aided VLC system for parallel vehicles.

$$
\mathrm{S}=\left[\begin{array}{c}
-\left(x_{s}+\frac{w_{m}}{2}+(j-1)\left(w_{m}+\Delta w_{m}\right)\right) \\
y_{s} \\
-\left(z_{s}+\frac{h_{m}}{2}+(i-1)\left(h_{m}+\Delta h_{m}\right)\right)
\end{array}\right],
$$

where $x_{s}$ represents the $\mathrm{x}$-coordinate and $z_{s}$ represents the $\mathrm{z}$ coordinate of $S$, as measured from the upper left corner of the mirror array.

The position vector of the receiver $\mathrm{D}$ can be expressed as follows:

$$
\mathrm{D}=\left[\begin{array}{c}
-\left(x_{d}+\frac{w_{m}}{2}+(j-1)\left(w_{m}+\Delta w_{m}\right)\right) \\
y_{d} \\
h_{d}-\left(z_{s}+\frac{h_{m}}{2}+(i-1)\left(h_{m}+\Delta h_{m}\right)\right)
\end{array}\right],
$$

where $x_{d}$ represents the $\mathrm{x}$-coordinate of $D$ as measured from the upper left corner of the mirror array and $h_{d}$ represents the $\mathrm{z}$-coordinate of $D$ as measured from $S$.

According to Snell's law of reflection, the angle between the incident direction and the mirror normal in an ideal specular reflection equals to the angle between the reflection direction and the normal. The position of the mirror must satisfy two conditions: first, the mirror must be within the reach of the light from the transmitter, and second, the mirror must have a suitable position and angle to ensure that the reflected light is within the range of FOV at the receiver.

As shown in Figure 2, the mirror in the IRS is first arranged via the clockwise rotation of the local $z$-axis with an angle $\beta_{i, j}$ and the local negative $x$-axis with an angle $\alpha_{i, j}$. A suitable position is obtained to enable the light to reach the receiver after being reflected by the mirror. The normal vector direction can be expressed as [34]

$$
\widehat{\mathrm{N}_{i, j}}=\frac{\widehat{\mathrm{R}_{i, j} \mathrm{~S}}+\widehat{\mathrm{R}_{\mathrm{i}, \mathrm{j}} \mathrm{D}}}{\sqrt{2+2 \widehat{\mathrm{R}, \mathrm{j}}^{\mathrm{T}} \mathrm{T} \widehat{\mathrm{R}_{\mathrm{i}, j} \mathrm{D}}}},
$$

where,

$$
\begin{gathered}
\widehat{\mathrm{R}_{\mathrm{i}, \mathrm{j}} \mathrm{S}}=\frac{\mathrm{S}-\mathrm{R}_{\mathrm{i}, \mathrm{j}}}{\left\|\mathrm{S}-\mathrm{R}_{\mathrm{i}, \mathrm{j}}\right\|_{2}}, \\
\widehat{\mathrm{R}_{\mathrm{i}, \mathrm{j}} \mathrm{D}}=\frac{\mathrm{D}-\mathrm{R}_{\mathrm{i}, \mathrm{j}}}{\left\|\mathrm{D}-\mathrm{R}_{\mathrm{i}, \mathrm{j}}\right\|_{2}}, \\
\|\bullet\|_{2}
\end{gathered}
$$

denote the $\ell_{2}$-norm and $(\bullet)^{\mathrm{T}}$ denotes the transpose operator. 
The relation between normal vector and rotation angle can be expressed as

$$
\widehat{N_{i, j}}=\left[\begin{array}{c}
\sin \left(\beta_{i, j}\right) \cos \left(\alpha_{i, j}\right) \\
\cos \left(\beta_{i, j}\right) \cos \left(\alpha_{i, j}\right) \\
\sin \left(\alpha_{i, j}\right)
\end{array}\right] .
$$

The size of the headlamp is much smaller than the distance of light transmission. Thus, it can be regarded as a point light source. The irradiance at the detector center contributed by all the mirrors can be expressed as [33]

$$
E=\sum_{i=1}^{n_{k}} \sum_{j=1}^{n_{l}} \frac{\rho(m+1) P_{i} \cos ^{m}\left(\theta_{R_{i, j}}^{S}\right)}{2 \pi\left(\left\|R_{i, j} D\right\|_{2}+\left\|R_{i, j} S\right\|_{2}\right)^{2}} \cos \left(\theta_{R_{i, j}}^{D}\right),
$$

where $P_{i}$ is the transmitted power and $\rho$ represents the mirror reflection efficiency. $m$ is the order of Lambertian emission [35] related to the half-power semiangle of LED $\Phi_{1 / 2}$ which can be expressed as $m=-\ln 2 / \ln \left(\cos \Phi_{1 / 2}\right)$.

According to Figure 2,

$$
\begin{aligned}
& \cos \left(\theta_{\mathrm{R}_{i, j}}^{\mathrm{S}}\right)= \mathrm{e}_{2}^{\mathrm{T}} \widehat{\mathrm{R}_{i, j} \mathrm{~S}}=\frac{\mathrm{e}_{2}^{\mathrm{T}}\left(\mathrm{S}-\mathrm{R}_{i, j}\right)}{\left\|\mathrm{S}-\mathrm{R}_{i, j}\right\|_{2}}, \\
& \cos \left(\theta_{\mathrm{R}_{\mathrm{i}, j}}^{\mathrm{D}}\right)= \mathrm{e}_{2}^{\mathrm{T}} \widehat{\mathrm{R}_{i, j} \mathrm{D}}=\frac{\mathrm{e}_{2}^{\mathrm{T}}\left(\mathrm{D}-\mathrm{R}_{i, j}\right)}{\left\|\mathrm{D}-\mathrm{R}_{i, j}\right\|_{2}}, \\
& \mathrm{e}_{2}^{\mathrm{T}}=[0,1,0] .
\end{aligned}
$$

According to the theory of VLC transmission [36], the DC gain of the channel can be obtained as

$$
H^{\mathrm{IRS}}(0)=\sum_{i=1}^{n_{k}} \sum_{j=1}^{n_{l}} \frac{\varpi T_{s}\left(\theta_{\mathrm{R}_{\mathrm{i}, \mathrm{j}}}^{\mathrm{D}}\right) A_{d} \rho(m+1) \cos ^{m}\left(\theta_{\mathrm{R}_{\mathrm{i}, \mathrm{j}}}^{\mathrm{S}}\right)}{2 \pi\left(\left\|\mathrm{R}_{\mathrm{i}, \mathrm{j}} \mathrm{D}\right\|_{2}+\left\|\mathrm{R}_{\mathrm{i}, \mathrm{j}} \mathrm{S}\right\|_{2}\right)^{2}} \cos \left(\theta_{\mathrm{R}_{\mathrm{i},}}^{\mathrm{D}}\right) g\left(\theta_{\mathrm{R}_{\mathrm{i}, \mathrm{j}}}^{\mathrm{D}}\right),
$$

where $\omega$ is the current-to-light conversion efficiency of the LED, $A_{d}$ is the detector physical area of the PD, and $T_{s}$ $\left(\theta_{R_{i, j}}^{D}\right)$ is the optical filter gain. $g\left(\theta_{R_{i, j}}^{D}\right)$ is the optical concentrator gain in the receiver, which can be given as

$$
g\left(\theta_{\mathrm{R}_{\mathrm{i}, \mathrm{j}}^{\mathrm{D}}}^{\mathrm{D}}\right)= \begin{cases}\frac{n^{2}}{\sin ^{2}\left(\Psi_{c}\right)} & 0 \leq \theta_{\mathrm{R}_{\mathrm{i}, \mathrm{j}}}^{\mathrm{D}} \leq \Psi_{c} \\ 0 & \theta_{\mathrm{R}_{\mathrm{i}, \mathrm{j}}}^{\mathrm{D}}>\Psi_{c}\end{cases}
$$

where $n$ is the refractive index and $\Psi_{c}$ is the width of FOV at a receiver.

The received signal can be expressed as

$$
y=\gamma H^{\mathrm{IRS}} x+w
$$

where $\gamma$ denotes the responsivity of a PD. $w$ is the additive white Gaussian noise obeying a distribution $\mathcal{N}\left(0, \sigma^{2}\right)$ with mean zero and variance $\sigma^{2}$, which can be concluded according to Reference [36].

The signal-to-noise ratio (SNR) can be expressed as follows:

$$
\mathrm{SNR}=\frac{\left(\gamma H^{\mathrm{IRS}}\right)^{2} \cdot P_{i}}{\sigma^{2}}
$$

For optical OOK modulation, the bit error rate (BER) is given by

$$
\mathrm{BER}=Q(\sqrt{\mathrm{SNR}})
$$

where,

$$
Q(x)=\frac{1}{\sqrt{2 \pi}} \int_{x}^{\infty} e^{-y^{2} / 2} d y .
$$

2.2. System Performance Analysis. According to Equations (8)-(11), the SNR is related to the number of mirrors and the distance of light transmission. The different numbers of mirrors and the distances of light transmission have different effects on the SNR.

2.2.1. The Influence of the Number of Mirrors. According to Equation (8), the DC gain of the channel increases continuously with the increase of the number of mirrors. The increase in the number of mirrors causes the size of the IRS to increase. But, the size of the IRS cannot be increased without a limit. Otherwise, the IRS is extended to the road, making the installation difficult and causing accidents quickly.

The received optical power and the noise increase with the number of mirrors increase according to Equations (8)-(11). In this case, the improvement of SNR gradually slows down with the BER. Therefore, the number of mirrors should be controlled to satisfy the communication requirements, and the system can also achieve the expected performance at a low cost.

2.2.2. The Influence of Optical Transmission Distance. According to Equation (8), the DC gain of the channel and the received optical power become larger with the smaller the optical transmission distance. The light is not enough to reach the IRS when the transmitter and the IRS are very close because of the limited radiation angle and half-power angle of the LED. Meanwhile, the FOV of the PD is limited, and no light is reflected to the receiver at this time. Thus, the received optical power is zero. Although the light from the transmitter can reach the receiver when the transmitter and the IRS are far away, the received power at this time decreases squarely as the distance increases, that is, the attenuation speed is fast. The optical power of the receiver is very small or even less than the noise power, resulting in low BER, which cannot satisfy the communication requirements.

The distance between the transmitter or the receiver and the IRS changes with the speed of the vehicle while the vehicle is moving. When the IRS is installed on the existing 
transportation infrastructures, the location of the IRS is fixed. To satisfy the communication requirements, multiple IRSs should be installed on the road, and their intervals must be controlled within a certain range. Regardless of the vehicle speed, the distance between the transmitter or receiver and the IRS is always within a suitable range. The distance of light transmission is not too close or too far, and the average BER can always satisfy the communication requirements.

\section{IRS Node Selection Scheme}

The analysis in Section 2.2 indicates that multiple IRSs should be installed to enable uninterrupted VLC between parallel vehicles, and their interval must be within an appropriate range. If the number of installed IRS is too large, additional transportation infrastructures must be provided, thereby increasing the cost. If the number of installed IRSs is insufficient, the distance between IRSs is too large, and the received power is too small that the communication may be interrupted. It is not conducive to communication continuity and may even cause traffic accidents. The application scenario is shown in Figure 3.

Assume that the number of mirrors in each IRS is the same and adjacent IRSs have the appropriate interval. In this case, one or even more IRSs can transmit the light from the transmitter to the receiver when multiple IRSs are installed. On the premise of the BER requirements, we face a problem of IRS node selection to maximize resource utilization.

3.1. Maximum Distance between Adjacent IRSs. The PD receives the light reflected from the multiple IRSs which can improve the received power. At this time, the total DC gain of the channel is

$$
H_{\text {total }}^{\mathrm{IRS}}(0)=\sum_{v=1}^{V} \sum_{i=1}^{n_{k}} \sum_{j=1}^{n_{l}} \frac{\varpi T_{s}\left(\theta_{\mathrm{R}_{\mathrm{i}, \mathrm{j}}^{\mathrm{v}}}^{\mathrm{D}}\right) A_{d} \rho(m+1) \cos ^{m}\left(\theta_{\mathrm{R}_{\mathrm{i}, \mathrm{j}}^{\mathrm{v}}}^{\mathrm{S}}\right)}{2 \pi\left(\left\|\mathrm{R}_{\mathrm{i}, \mathrm{j}}^{\mathrm{v}} \mathrm{D}\right\|_{2}+\left\|\mathrm{R}_{\mathrm{i}, \mathrm{j}}^{\mathrm{v}} \mathrm{S}\right\|_{2}\right)^{2}} \cos \left(\theta_{\mathrm{R}_{\mathrm{i}, \mathrm{j}}^{\mathrm{v}}}^{\mathrm{D}}\right) g\left(\theta_{\mathrm{R}_{\mathrm{i}, \mathrm{j}}^{\mathrm{v}}}^{\mathrm{D}}\right),
$$

where $v$ is a positive integer which is the serial number of the IRSs. $V$ is the total number of the IRSs.

Assume that the distance between adjacent IRS is $D_{\text {IRS }}$. The relationship between $R_{i, j}^{v+1}$ and $R_{i, j}^{v}$ can be expressed as

$$
\mathrm{e}_{2}^{\mathrm{T}} \mathrm{R}_{\mathrm{i}, \mathrm{j}}^{\mathrm{v}+1}=\mathrm{e}_{2}^{\mathrm{T}} \mathrm{R}_{\mathrm{i}, \mathrm{j}}^{\mathrm{v}}+D_{\mathrm{IRS}}
$$

The total received signal can be expressed as

$$
y=\gamma H_{\text {total }}^{\mathrm{IRS}}(0) x+w
$$

The total SNR can be expressed as

$$
\mathrm{SNR}_{\text {total }}=\frac{\left(\gamma H_{\text {total }}^{\mathrm{IRS}}(0)\right)^{2} P_{i}}{\sigma^{2}}
$$

The total BER for optical OOK modulation can be expressed as

$$
\mathrm{BER}_{\text {total }}=Q\left(\sqrt{\mathrm{SNR}_{\text {total }}}\right)
$$

In the $\mathrm{OOK}$ modulation, $\mathrm{SNR}$ requires $13.6 \mathrm{~dB}$ to achieve $\mathrm{BER}=10^{-6}$. Then, the maximum distance $D_{\text {IRS, max }}$ between adjacent IRSs under this BER can be calculated.

3.2. IRS Node Selection Scheme. For parallel vehicles, the distance between the transmitter or receiver and the IRS on the $y$-axis is equal. When the vehicle is driving in the area of any two adjacent IRS, it faces the same problem of IRS node selection. Thus, we choose one of the areas between two adjacent IRSs as an example.

We assume that a total of $V$ IRSs are installed, and each IRS contains the same number of mirrors. At this time, the received power corresponds to the distance of the light transmission one by one. We propose the NNIS algorithm for selecting IRS nodes to ensure that the least IRSs are used. The specific steps are as follows.

(1) Assume that $v(1 \leq v \leq V)$ represents the number of IRSs. The numbering rules are arranged from small to large along the direction of the vehicle according to the distance between the IRS and the transmitter. The vehicles are traveling in the area between two adjacent IRSs. When the distance between the receiver and the nearest IRS (that is, $\left.\operatorname{IRS}_{1}\right)$ is $D_{o}\left(0 \leq D_{o} \leq D_{\text {IRS }}\right)$ on $y$-axis, then the received power $P_{t, v}$ can be calculated at different optical transmission distances $D_{o}+$ $(v-1) \cdot D_{\mathrm{IRS}}$ according to Equations (8)-(11)

(2) Sort $P_{t, v}$ in descending order to obtain $P_{t, v}^{\prime}$, where $v^{\prime}\left(1 \leq v^{\prime} \leq V\right)$ represents the number of $P_{t, v^{\prime}}^{\prime} . P_{t, 1}^{\prime}$ is the maximum value of $P_{t, v^{\prime}}$

(3) Calculate the received power $P_{t, 0}$ when $S N R=13.6$ $\mathrm{dB}$ according to Equation (11)

(4) If $P_{t, 1}^{\prime} \geq P_{t, 0}$, only one IRS is required to work. Find the smallest value $P_{t, v^{\prime}}^{\prime}$ greater than $P_{t, 0}$ in $\left\{P_{t, v^{\prime}}^{\prime} \mid 1\right.$ $\left.\leq v^{\prime} \leq V\right\}$ and the corresponding IRS with this value. Only this IRS can be used to satisfy the SNR requirements. The average received power at this time is $P_{t}=\left\{\min P_{t, v^{\prime}}^{\prime} \mid P_{t, v^{\prime}}^{\prime} \geq P_{t, 0}, 1 \leq v^{\prime} \leq V\right\}$

(5) If $P_{t, 1}^{\prime}+P_{t, 2}^{\prime}+\cdots+P_{t, q}^{\prime}<P_{t, 0} \leq P_{t, 1}^{\prime}+P_{t, 2}^{\prime}+\cdots+P_{t, q}^{\prime}+P_{t, q+1}^{\prime}$, $1 \leq q \leq V-1$, the first $(q+1)$ IRSs corresponding to $P_{t, v^{\prime}}^{\prime}$ must work at the same time to satisfy the SNR requirement. The received power according to Equations (14)-(17) at this time is $\mathrm{P}_{\mathrm{t}}=\left\{\mathrm{P}_{\mathrm{t}, 1}^{\prime}+\mathrm{P}_{\mathrm{t}, 2}^{\prime}+\cdots+\mathrm{P}_{\mathrm{t}, \mathrm{q}}^{\prime}+\right.$ $\mathrm{P}_{\mathrm{t}, \mathrm{q}+1}^{\prime} \mid \mathrm{P}_{\mathrm{t}, 1}^{\prime}+\mathrm{P}_{\mathrm{t}, 2}^{\prime}+\cdots+\mathrm{P}_{\mathrm{t}, \mathrm{q}}^{\prime}<\mathrm{P}_{\mathrm{t}, 0} \leq \mathrm{P}_{\mathrm{t}, 1}^{\prime}+\mathrm{P}_{\mathrm{t}, 2}^{\prime}+\cdots+\mathrm{P}_{\mathrm{t}, \mathrm{q}}^{\prime}+$ $\left.\mathrm{P}_{\mathrm{t}, \mathrm{q}+1}^{\prime}, 1 \leq \mathrm{q} \leq \mathrm{V}-1\right\}$

(6) When the parallel vehicles move to the next adjacent IRS area, repeat the above steps

The NNIS algorithm is shown in Algorithm 1. 


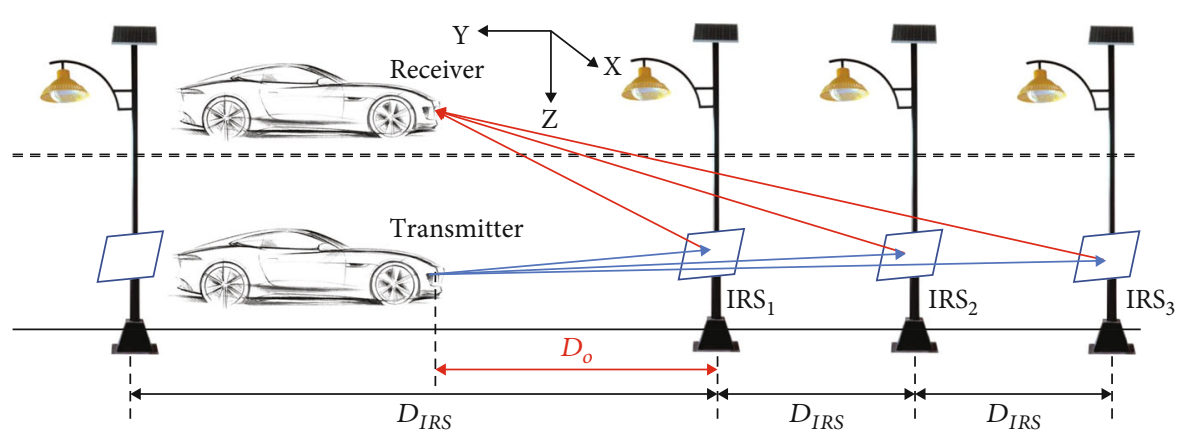

FIGURE 3: The application scenario of multiple IRS-aided V2V VLC system.

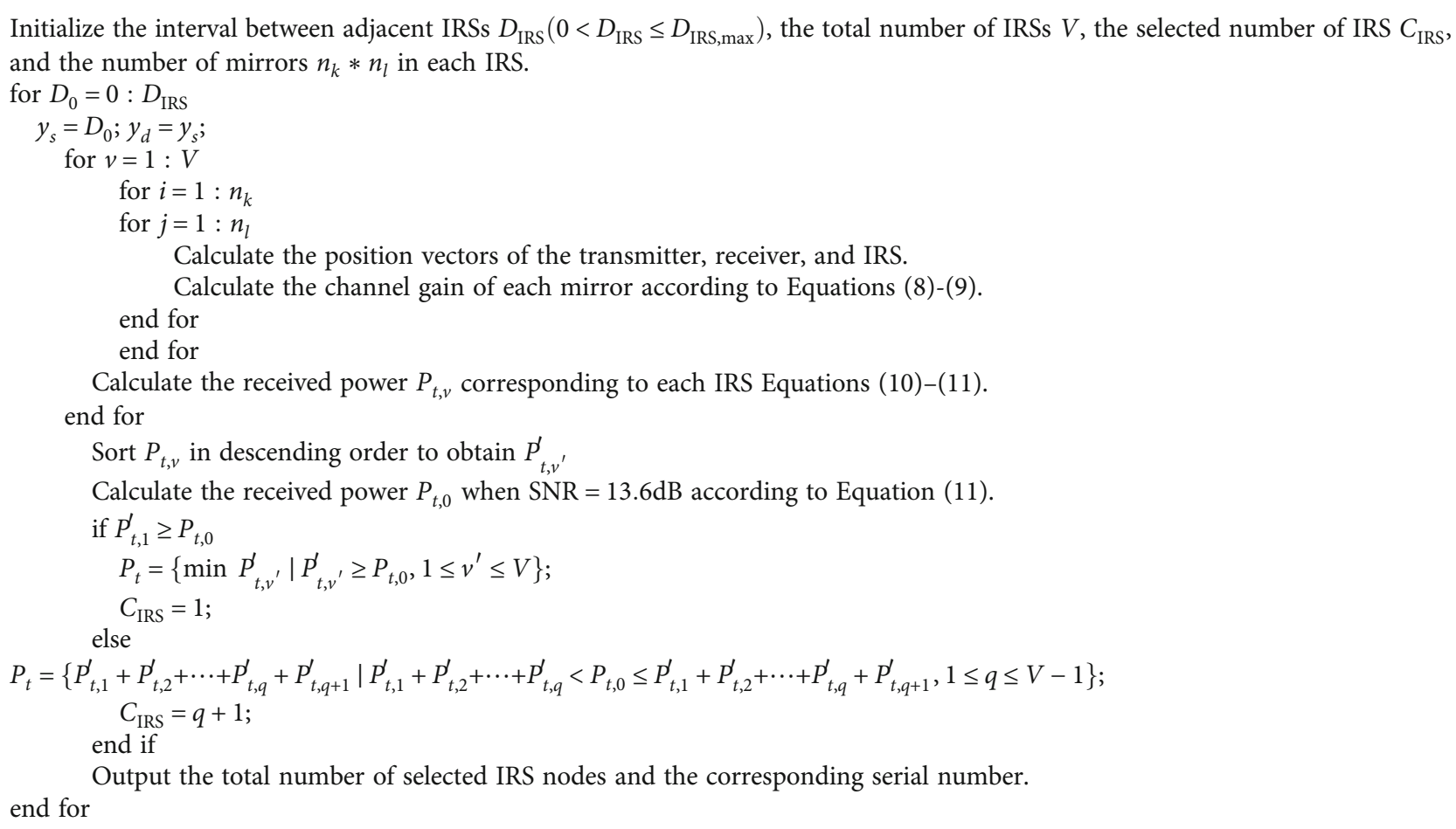

Algorithm 1: The NNIS algorithm.

Under the premise of satisfying the BER requirements, the least IRS nodes can be selected to aid the VLC using the NNIS algorithm and realize uninterrupted communication. The IRS occupancy rate can be reduced, and the free IRSs can be utilized to VLC for other vehicles.

\section{Numerical Results}

In this section, we present the numerical results to evaluate the performance of the IRS-aided VLC system for parallel vehicles. The main parameters are given first, and then, the influence of the number of mirrors and the position of the IRSs are analyzed. Finally, the IRS node selection scheme is verified.
4.1. Simulation Environment. The main system parameters are summarized in Table 1. Assume that the vehicles are driving along the centerline of the lane with a width of 3.5 meters. The lamp mounting height for high-beam headlamps is $0.62 \mathrm{~m}$, and lamp separation is $1.12 \mathrm{~m}$. The transmitted power is $30 \mathrm{~W}$, and the half-power semiangle is $60 \mathrm{deg}$. As the receiver, $\mathrm{PD}$ is installed in the middle of two headlamps. The detector physical area is $1.0 \mathrm{~cm}^{2}$, and the FOV is $35 \mathrm{deg}$. IRSs are installed on the street light poles, and the height of the center is consistent with that of the headlamps. The size of each mirror in IRS is $0.02 \times 0.02 \mathrm{~m}^{2}$, and the spacing between mirrors is zero. The mirror reflection efficiency is 0.8. The modulation mode is optical OOK modulation. 
TABLE 1: Main system parameters.

\begin{tabular}{lcc}
\hline Symbol & Parameters & Value \\
\hline$z_{m}$ & Height of the IRS center & $0.62 \mathrm{~m}$ \\
$\left(w_{m}, h_{m}\right)$ & Size of a mirror & $(0.02,0.02) \mathrm{m}$ \\
$P_{\text {LED }}$ & Transmitted optical power & $30 \mathrm{~W}$ \\
$\Phi_{1 / 2}$ & Half-power semiangle of a LED & $60 \mathrm{deg}$. \\
$\Psi_{c}$ & FOV of a PD & $35 \mathrm{deg}$. \\
$A_{d}$ & Detector physical area of the PD & $1.0 \mathrm{~cm}^{2}$ \\
$T_{s}$ & Optical filter gain & 1.0 \\
$n$ & Refractive index & 1.5 \\
$\gamma$ & Responsivity of a PD & $0.54 \mathrm{~A} / \mathrm{W}$ \\
$\rho$ & Mirror reflection efficiency & 0.8 \\
$\omega$ & Current-to-light conversion efficiency & $0.44 \mathrm{~W} / \mathrm{A}$ \\
\hline
\end{tabular}

We can obtain the positions of the transmitter and receiver depending on the above conditions.

$$
x_{s}=\left(\frac{3.5}{2}-\frac{1.12}{2}\right)=1.19, z_{s}=-\left(w_{m} \cdot \frac{n_{l}}{2}\right)=-\left(0.05 * \frac{n_{l}}{2}\right) .
$$

$y_{s}$ is constantly changing with the movement of the vehicles.

$$
x_{d}=\left(3.5+\frac{3.5}{2}\right)=-5.25, y_{d}=y_{s}, z_{d}=0 .
$$

\subsection{Numerical Results}

4.2.1. SNR Comparison with Different Numbers of Mirrors in the IRS. When the number of mirrors in IRS is different, the paths of light transmission are different, bringing different changes to the received power and SNR. Figure 4 shows the change of SNR caused by different numbers of mirrors in the range from 0 to 100 meters with a $1 \mathrm{~m}$ interval for $y_{d}$ when only one IRS exists.

As can be seen in Figure 4, the SNR equals zero regardless of the number of mirrors when $0 \leq y_{d} \leq 7 \mathrm{~m}$. The reason is that the FOV of the PD is limited, and the incident angle within this distance range is larger than the FOV. According to Equation (9), the optical concentrator gain is zero, so the received power is zero. When $8 \mathrm{~m} \leq y_{d} \leq 100 \mathrm{~m}$, the SNR monotonically decreases with the increase of $y_{d}$. When $y_{d}$ $\leq 56 \mathrm{~m}$, all the SNRs in the simulation are less than $13.6 \mathrm{~dB}$. According to Equation (8), the increase of $y_{d}$ causes the continuous increase of $\left\|R_{i, j} D\right\|_{2}$ and $\left\|R_{i, j} S\right\|_{2}$, resulting in the decrease of the received power and SNR.

At the same $y_{d}$, the SNR continues to increase with the increase of the number of mirrors. The reason is that light can be transmitted through more different paths to the receiver, thereby increasing the received power and the SNR continuously. The rate of SNR improvement gradually decreases with the increase of the number of mirrors. Because the increase in the number of mirrors is equivalent to adding a circle of mirrors to the existing IRS edge, the

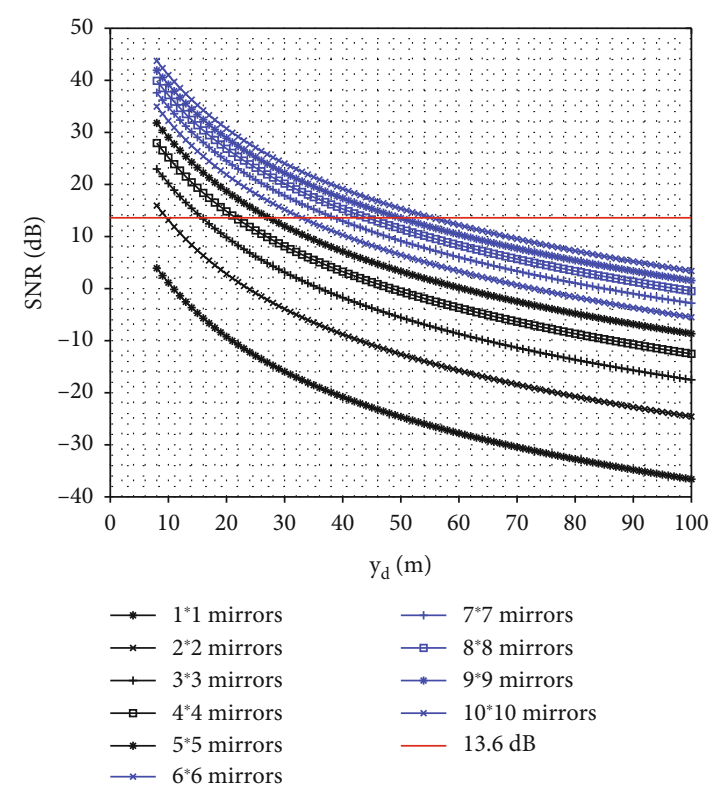

FIGURE 4: SNRs with different numbers of mirrors in the IRS.

central position of the IRS is fixed, and the light transmitted distance of a newly added mirror is larger than that of the mirror in the original IRS. The received optical power is small, and the total received power increases slowly.

If the IRS includes too many mirrors, it will occupy the road and easily cause traffic accidents. The number of mirrors and the deployment location must be simultaneously considered to avoid the SNR less than $13.6 \mathrm{~dB}$. In this study, the number of mirrors in the IRS is selected as $6 \times 6$ following the above analysis. In this case, the SNR is higher than $13.6 \mathrm{~dB}$ when $8 \mathrm{~m} \leq y_{d} \leq 32 \mathrm{~m}$. For urban roads, the spacing of traffic infrastructure (such as street light poles) is generally less than $32 \mathrm{~m}$. When $y_{d} \leq 7 \mathrm{~m}$, one or more IRSs at a distance can be considered to aid VLC together which can make SNR larger than zero. Thus, a sufficient number of IRSs can work simultaneously to make the SNR achieve the communication requirements.

4.2.2. The Maximum Distance between Adjacent IRS. According to the analysis in Section 4.2.1, multiple IRSs should work together to ensure that the SNR is greater than $13.6 \mathrm{~dB}$ when $y_{d}>32 \mathrm{~m}$. We use the exhaustive method to find $D_{\text {IRS, max }}$ under the premise of satisfying the SNR requirements. The change of SNR brought by the different numbers of IRS $N_{\text {IRS }}$ is analyzed under a certain $D_{\text {IRS }}$ first. Then, the change of SNR brought by different $D_{\text {IRS }}$ is analyzed under a certain $N_{\text {IRS }}$.

(1) SNR versus Different $N_{\text {IRS }}$ with a Certain $D_{\text {IRS. Figure } 5}$ shows the SNR comparison of different $N_{\text {IRS }}$ when $D_{\text {IRS }}=5$ $\mathrm{m}, 10 \mathrm{~m}, 20 \mathrm{~m}$, and $30 \mathrm{~m}$, respectively. When $N_{\text {IRS }}=1$, only one nearest neighbor IRS works. When $D_{\text {IRS }}=5 \mathrm{~m}$, since the light reflected by IRS cannot be received because of the FOV limitation of PD, the SNR is zero. Similarly, the SNR is zero with $D_{\text {IRS }}=10 \mathrm{~m}, 20 \mathrm{~m}$, and $30 \mathrm{~m}$ when $0 \mathrm{~m} \leq D_{\text {IRS }} \leq 7$ 


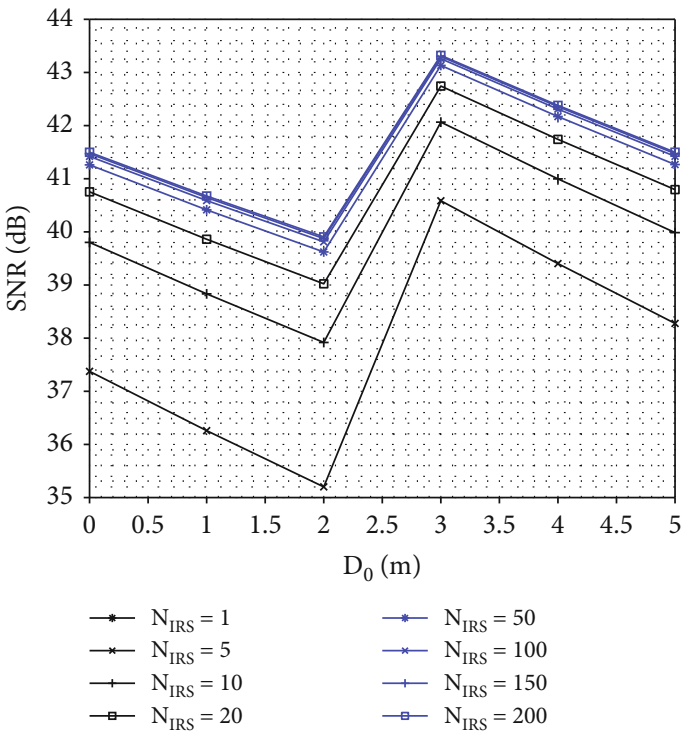

(a)

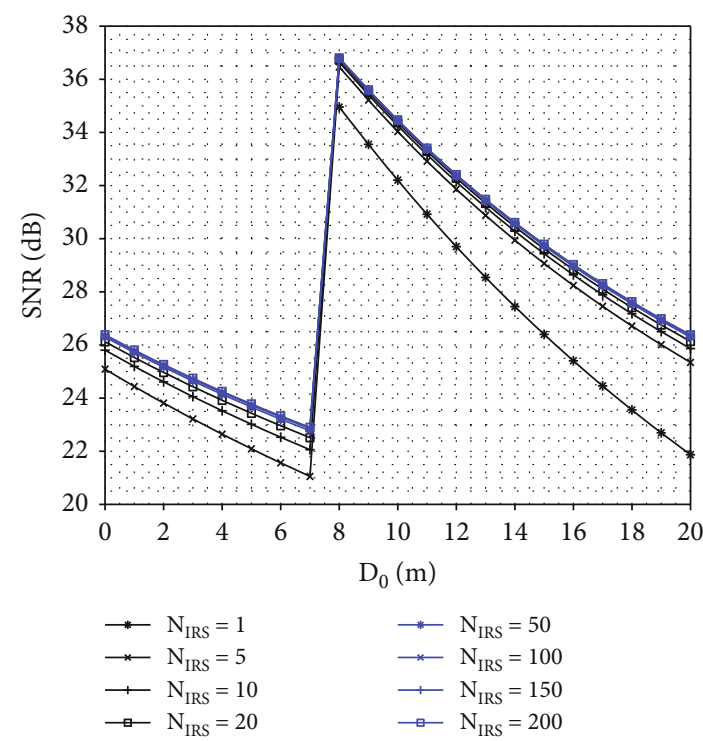

(c)

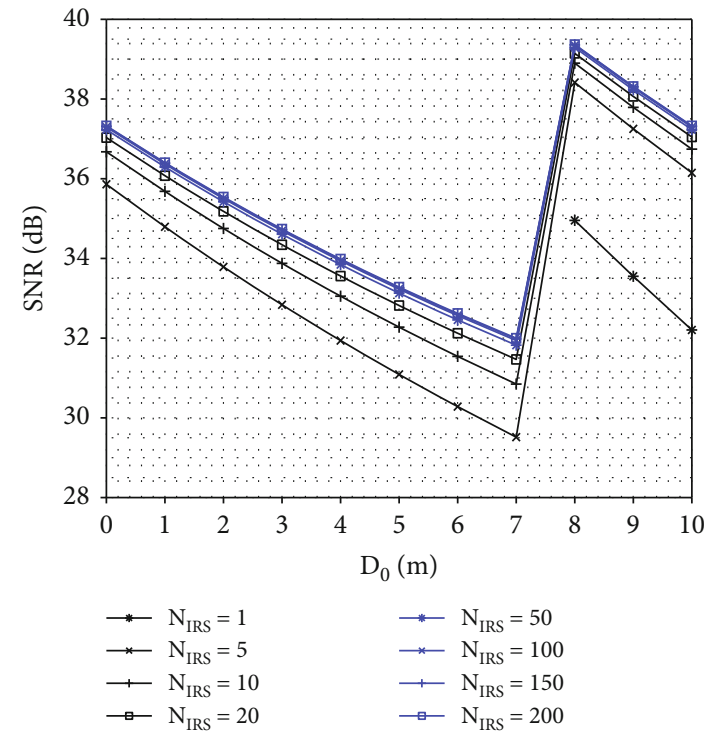

(b)
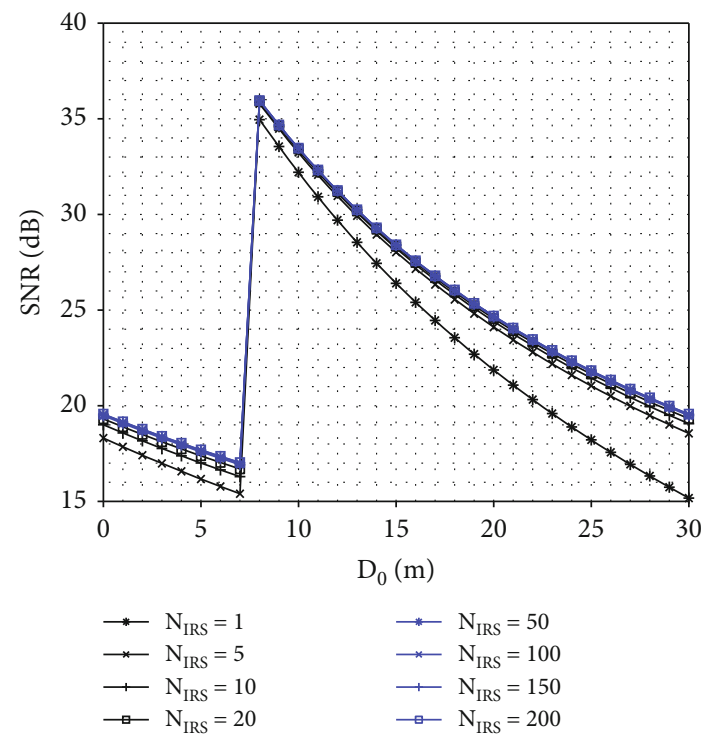

(d)

FIGURE 5: SNR comparison with different $N_{\text {IRS }}$ when (a) $D_{\text {IRS }}=5 \mathrm{~m}$, (b) $D_{\text {IRS }}=10 \mathrm{~m}$, (c) $D_{\text {IRS }}=20 \mathrm{~m}$, and (d) $D_{\text {IRS }}=30 \mathrm{~m}$.

m. Therefore, communication may be interrupted when $N_{\text {IRS }}=1$.

When $N_{\text {IRS }}=5,10,20,50,100,150$, and 200, with the intervals of PD and different IRSs increasing, the incident angle of the reflected light from multiple IRSs is less than FOV, avoiding the zero SNR. At this time, the SNR is higher than $13.6 \mathrm{~dB}$ when multiple IRSs work together.

However, the increase rate of the SNR decreases with the increase of $N_{\text {IRS. }}$. The reason is that the received power decreases faster with the distance increase. So, the IRSs installed farther away contribute little to the total SNR. The increase of $N_{\text {IRS }}$ also implies that more infrastructures are required to install IRSs, resulting in increasing costs of communication. Figure 5 shows that the SNRs of $N_{\text {IRS }}=50$
, 100, 150, and 200 are almost the same when $D_{\text {IRS }}=10 \mathrm{~m}$, $20 \mathrm{~m}$, and $30 \mathrm{~m}$. Therefore, this study selects $N_{\text {IRS }}=50$ to save resources and reduce costs.

(2) SNR versus Different $D_{\text {IRS }}$ with a Certain $N_{\text {IRS }}$. We use the exhaustive method to calculate $D_{\text {IRS,max }}$. Figure 6 shows the comparison of SNR with different $D_{\text {IRS }}$ when $N_{\text {IRS }}=50$.

As can be seen from Figure 6, the SNR becomes larger with the $D_{\text {IRS }}$ decrease. The reason is that the smaller $D_{\text {IRS }}$ implies the smaller optical transmission distance and larger received power at the same location. In the adjacent IRS area, SNR has a large jump between $D_{0}=7 \mathrm{~m}$ and $D_{0}=8 \mathrm{~m}$, which is also the transition stage from the incident angle of the nearest IRS greater than FOV to that less than FOV. 


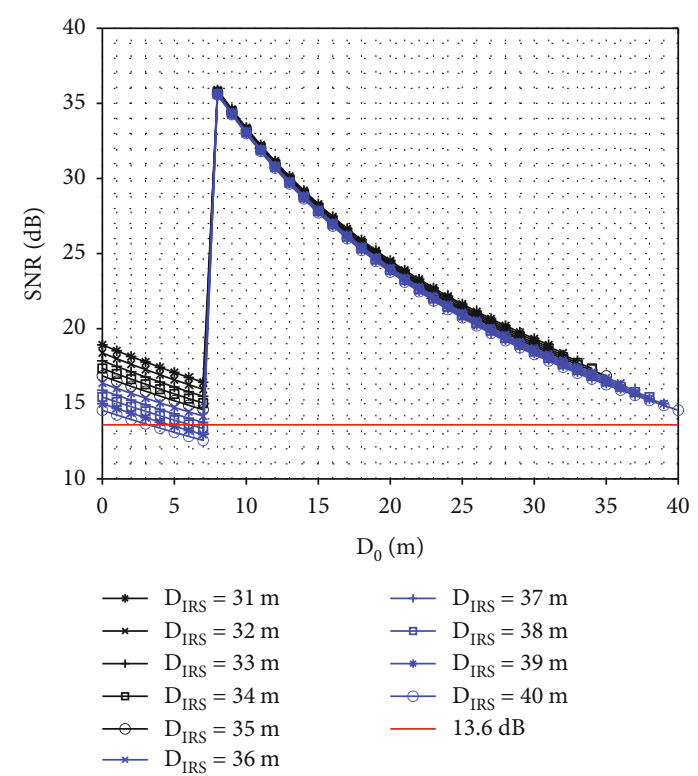

FIgURE 6: SNR comparison with different $D_{\text {IRS }}$ when $N_{\text {IRS }}=50$.

When $D_{0}=8 \mathrm{~m}$, the incident angle of the IRSs starts to be smaller than the FOV. At this time, the received power contribution of the nearest IRS is the largest. The SNR when 0 $\mathrm{m} \leq D_{0} \leq 7 \mathrm{~m}$ is lower than that when $8 \mathrm{~m}<D_{0}<D_{\text {IRS. The }}$ reason is that the incident angle of the nearest IRS is smaller than FOV when $8 \mathrm{~m}<D_{0}<D_{\text {IRS }}$, thereby improving the received power and SNR compared with those in other IRSs.

The SNR is larger than $13.6 \mathrm{~dB}$ in the two adjacent IRS areas when $D_{\text {IRS }}=31 \mathrm{~m}, 32 \mathrm{~m}, 33 \mathrm{~m}, 34 \mathrm{~m}, 35 \mathrm{~m}, 36 \mathrm{~m}$, and 37 $m$. When $D_{\text {IRS }}=38 \mathrm{~m}$, the SNRs corresponding to $D_{0}=7 \mathrm{~m}$ are less than $13.6 \mathrm{~dB}$. When $D_{\text {IRS }}=39 \mathrm{~m}$, the SNRs corresponding to $D_{0}=5 \mathrm{~m}, 6 \mathrm{~m}$, and $7 \mathrm{~m}$ are less than $13.6 \mathrm{~dB}$. When $D_{\text {IRS }}=40 \mathrm{~m}$, the SNRs corresponding to $D_{0}=4 \mathrm{~m}, 5 \mathrm{~m}$ , $6 \mathrm{~m}$, and $7 \mathrm{~m}$ are less than $13.6 \mathrm{~dB}$. In other words, the SNRs are less than $13.6 \mathrm{~dB}$ that occur when $D_{\text {IRS }} \geq 38 \mathrm{~m}$. The course of the situation continues with the increase of $D_{\text {IRS }}$. When $D_{\text {IRS }} \leq 37 \mathrm{~m}$, no SNR less than $13.6 \mathrm{~dB}$ exists. Therefore, $D_{\text {IRS, } \max }=37 \mathrm{~m}$ when $N_{\text {IRS }}=50$.

4.2.3. Experiment of IRS Node Selection. According to the analysis in Section 4.2.2, when $D_{\text {IRS }} \leq 37 \mathrm{~m}$, regardless of where the vehicle is driving, the $\mathrm{SNR}$ is larger than $13.6 \mathrm{~dB}$ using one or more IRSs. This scenario requires node selection for IRSs. We verify the effectiveness of the IRS node selection scheme according to the NNIS algorithm. In urban roads, the interval between street light poles is determined by the height of the lamp pole, the power of the lamp, and the width of the road, which is generally from $15 \mathrm{~m}$ to $50 \mathrm{~m}$. Firstly, we select $D_{\text {IRS }}$ $=20 \mathrm{~m}$, which is the normal interval between street light poles in urban roads. Figure 7 shows the SNR performance of the NNIS algorithm when $D_{\text {IRS }}=20 \mathrm{~m}$.

Figure 7 shows that when only IRS $_{1}$ works, the SNR is equal to zero because of the FOV limitation when $0 \leq D_{0} \leq$ $7 \mathrm{~m}$. The SNR is no longer limited to FOV and larger than $13.6 \mathrm{~dB}$ when $8 \mathrm{~m} \leq D_{0} \leq 20 \mathrm{~m}$. When only IRS $_{2}$ works, the

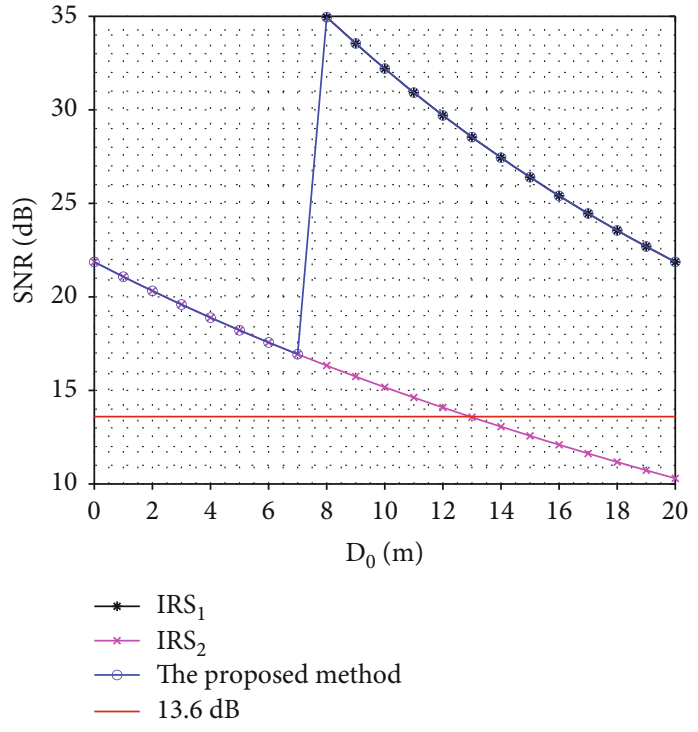

FIGURE 7: SNR performance of the NNIS algorithm when $D_{\text {IRS }}=20 \mathrm{~m}$

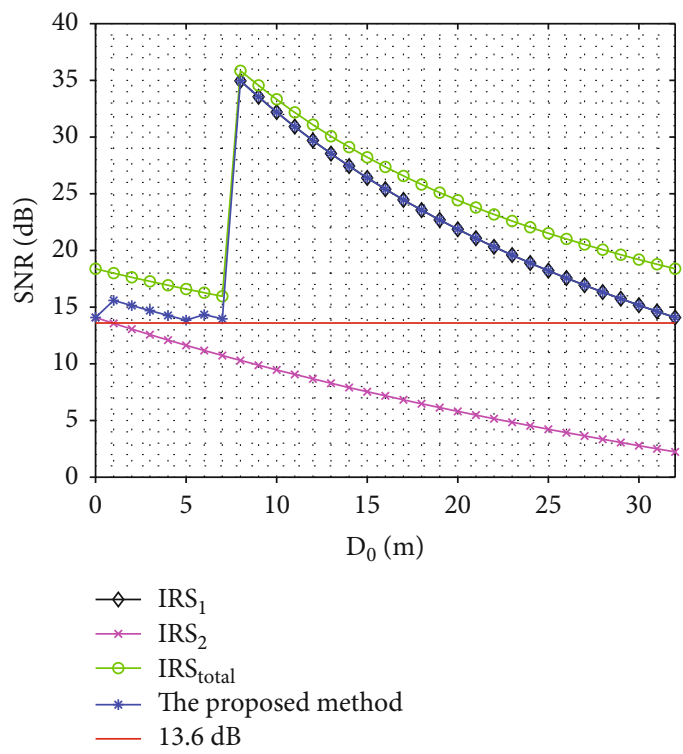

FIGURE 8: SNR performance of the NNIS algorithm when $D_{\text {IRS }}=32 \mathrm{~m}$

received power is not limited to FOV, and the SNR is greater than zero with the increase of distance. As $D_{0}$ increases, SNR decreases continuously. SNR is less than $13.6 \mathrm{~dB}$ when $13 \mathrm{~m}$ $\leq D_{0} \leq 20 \mathrm{~m}$.

The IRSs must be selected to ensure that SNR is greater than $13.6 \mathrm{~dB}$ in the range of $0 \leq D_{0} \leq 20 \mathrm{~m}$. According to the NNIS algorithm, we select IRS 2 when $0 \leq D_{0} \leq 7 \mathrm{~m}$ and select IRS 1 when $8 \mathrm{~m} \leq D_{0} \leq 20 \mathrm{~m}$. Therefore, when $D_{\text {IRS }}=$ $20 \mathrm{~m}$, only one IRS node selection problem is involved.

The SNR is larger than $13.6 \mathrm{~dB}$ when $8 \mathrm{~m} \leq y_{d} \leq 32 \mathrm{~m}$

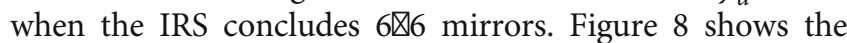
SNR performance of the NNIS algorithm when $D_{\text {IRS }}=32 \mathrm{~m}$. 


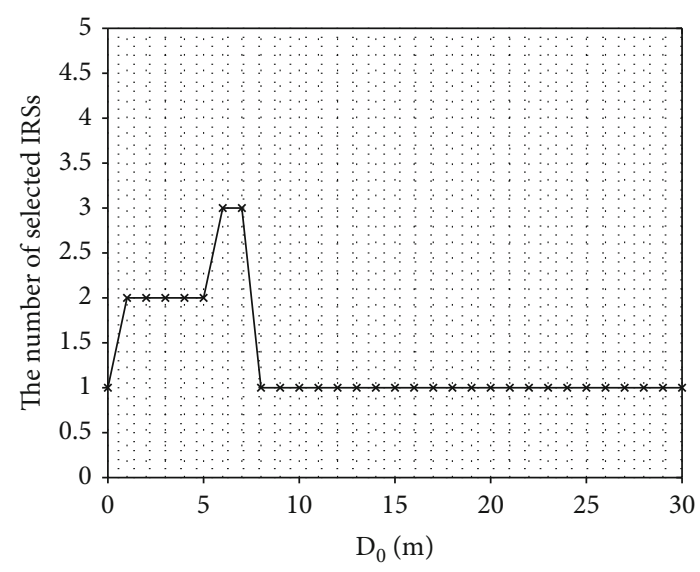

Figure 9: Number of IRS used with different values of $D_{0}$

As can be seen from Figure 8, all the values of SNRs using the NNIS algorithm are larger than $13.6 \mathrm{~dB}$. This scenario involves one or more IRS selection issues. Only IRS 2 is required when $D_{0}=0 \mathrm{~m}$. When $8 \mathrm{~m} \leq D_{0} \leq 32 \mathrm{~m}$, only IRS $_{1}$ is required. Neither IRS ${ }_{1}$ nor IRS ${ }_{2}$ can satisfy the SNR requirement, and more IRSs should be used when $1 \mathrm{~m} \leq D_{0}$ $\leq 7 \mathrm{~m}$. Figure 9 shows the number of IRSs used with different values of $D_{0}$.

As can be seen from Figure 9, two IRSs are selected to work together when $1 \mathrm{~m} \leq D_{0} \leq 5 \mathrm{~m}$ and three IRSs are selected to work together when $6 \mathrm{~m} \leq D_{0} \leq 7 \mathrm{~m}$. Although the SNR performance of the NNIS algorithm is inferior to that of using all IRSs, the fewest IRSs working simultaneously ensure that the SNR can satisfy requirements using the NNIS algorithm. The number of IRSs used in VLC is reduced and enables the free IRSs to aid the VLC for other vehicles, thereby greatly improving the efficiency of IRS resources.

\section{Conclusion}

In this study, the mirror array-based IRS-aided VLC system for parallel vehicles is designed to solve the problem of VLC interruption. Then, the SNR, the BER, the influence of the number of mirrors, and the distance between adjacent IRSs of the system are analyzed. The calculation method of the maximum distance between adjacent IRS satisfying SNR requirements is given. Finally, the NNIS algorithm for IRS node selection is proposed. The numerical results show that when the number of mirrors in the IRS is $6 \times 6$, the maximum distance between adjacent IRSs satisfying the SNR requirement is $37 \mathrm{~m}$. The NNIS algorithm is used to calculate the fewest number of selected IRSs. The required SNR can be satisfied with merely three IRSs working simultaneously when the adjacent IRSs interval is $32 \mathrm{~m}$. The remaining IRSs can be used to aid VLC for other vehicles, and the resource utilization efficiency of the IRSs can be improved.

The number of mirrors in each IRS and the distance between adjacent IRSs are presumably fixed to facilitate the analysis in this study. Since each mirror in the IRS can be intelligently controlled, the mirrors in the IRS can be considered to aid VLC for multiple vehicles under the premise of satisfying the SNR requirement in future. In addition, with the number of transportation infrastructures along the road increasing, the distances between them are changed which facilitates the deployment of IRSs. Thus, the issue of the IRS node selection should be considered with different distances between adjacent IRSs to improve the system efficiency further.

\section{Data Availability}

The data used to support the findings of this study are available from the submitting author upon request.

\section{Conflicts of Interest}

The authors declare that there is no conflict of interest regarding the publication of this paper.

\section{Acknowledgments}

This work was supported in part by the National Natural Science Foundation of China under Grants 61966007 and 61961007; the Basic Ability Improvement Project of Young and Middle-Aged Teachers in Guangxi Universities under Grant number 2021KY0217; the Key Laboratory of Cognitive Radio and Information Processing, Ministry of Education under Grants CRKL170110, CRKL180201, and CRKL180106; and the School of Information and Communication, Guangxi Key Laboratory of Wireless Wideband Communication and Signal Processing, Guilin University of Electronic Technology, China (GXKL0619204 and GXKL06200116).

\section{References}

[1] D. Hahn, A. Munir, and V. Behzadan, "Security and privacy issues in intelligent transportation systems: classification and challenges," IEEE Intelligent Transportation Systems Magazine, vol. 13, no. 1, pp. 181-196, 2021.

[2] Z. Lv, R. Lou, and A. K. Singh, "AI empowered communication systems for intelligent transportation systems," IEEE Transactions on Intelligent Transportation Systems, vol. 22, no. 7, pp. 4579-4587, 2021.

[3] L. Zhu, H. Liang, H. Wang, B. Ning, and T. Tang, "Joint security and train control design in blockchain empowered CBTC system," IEEE Internet of Things Journal, 2021.

[4] M. B. Mollah, J. Zhao, D. Niyato et al., "Blockchain for the internet of vehicles towards intelligent transportation systems: a survey," IEEE Internet of Things Journal, vol. 8, no. 6, pp. 4157-4185, 2021.

[5] A. Lamssaggad, N. Benamar, A. S. Hafid, and M. Msahli, "A survey on the current security landscape of intelligent transportation systems," IEEE Access, vol. 9, pp. 9180-9208, 2021.

[6] L. Zhu, Y. Li, F. Richard Yu, B. Ning, T. Tang, and X. X. Wang, "Cross-layer defense methods for jamming-resistant CBTC systems," IEEE Transactions on Intelligent Transportation Systems, vol. 22, no. 11, pp. 7266-7278, 2021.

[7] P. Bagga, A. K. Das, M. Wazid, J. J. P. C. Rodrigues, K.-K. R. Choo, and Y. Park, "On the design of mutual authentication and key agreement protocol in internet of vehicles-enabled intelligent transportation system," IEEE Transactions on Vehicular Technology, vol. 70, no. 2, pp. 1736-1751, 2021. 
[8] Y. Li, L. Zhu, H. Wang, F. R. Yu, and S. Liu, "A cross-layer defense scheme for edge intelligence-enabled CBTC systems against MitM attacks," IEEE Transactions on Intelligent Transportation Systems, vol. 22, no. 4, pp. 2286-2298, 2021.

[9] T. do Vale Saraiva, C. A. Campos, R. dos Reis Fontes, C. E. Rothenberg, S. Sorour, and S. Valaee, “An application-driven framework for intelligent transportation systems using $5 \mathrm{G}$ network slicing," IEEE Transactions on Intelligent Transportation Systems, vol. 22, no. 8, pp. 5247-5260, 2021.

[10] A. Sharma, A. Prajapati, and P. Pinho, "Interference-aware antenna synthesis for enhanced coverage in intelligent transportation system," IEEE Transactions on Vehicular Technology, vol. 70, no. 8, pp. 7803-7811, 2021.

[11] L. Y. Wei, C. W. Chow, Y. Liu, and C. H. Yeh, "Multi-Gbit/s phosphor-based white-light and blue-filter-free visible light communication and lighting system with practical transmission distance," Optics Express, vol. 28, no. 5, pp. 7375-7381, 2020.

[12] B. Bechadergue, L. Chassagne, and H. Y. Guan, "Experimental comparison of pulse-amplitude and spatial modulations for vehicle-to-vehicle visible light communication in platoon configurations," Optics Express, vol. 25, no. 20, pp. 24790-24802, 2017.

[13] C. Wang, G. Li, F. Hu et al., "Visible light communication for vehicle to everything beyond $1 \mathrm{~Gb} / \mathrm{s}$ based on an LED car headlight and a $2 \times 2$ PIN array," Chinese Optics Letters, vol. 18, no. 11, p. 110602, 2020.

[14] H. B. Eldeeb, E. Eso, E. A. Jarchlo et al., "Vehicular VLC: a ray tracing study based on measured radiation patterns of commercial taillights," IEEE Photonics Technology Letters, vol. 33, no. 16, pp. 904-907, 2021.

[15] O. Narmanlioglu, B. Turan, S. C. Ergen, and M. Uysal, “Cooperative MIMO-OFDM based inter-vehicular visible light communication using brake lights," Computer Communications, vol. 120, pp. 138-146, 2018.

[16] M. A. Atta and A. Bermak, "A 160 m visible light communication link using hybrid undersampled phase-frequency shift onoff keying and CMOS image sensor," Optics Express, vol. 27, no. 3, pp. 2478-2487, 2019.

[17] J. He and B. A. Zhou, "Vehicle positioning scheme based on visible light communication using a CMOS camera," Optics Express, vol. 29, no. 17, pp. 27278-27290, 2021.

[18] W. Zhao, M. Kamezaki, K. Yamaguchi, M. Konno, A. Onuki, and S. Sugano, "Investigation on image signal receiving performance of photodiodes and solar panel detectors in an underground facility visible light communication system," Optics Express, vol. 29, no. 2, pp. 692-705, 2021.

[19] D. L. Msongaleli and K. Kucuk, "Optimal resource utilisation algorithm for visible light communication-based vehicular ad-hoc networks," IET Intelligent Transport Systems, vol. 14, no. 2, pp. 65-72, 2020.

[20] L. Qiao, X. Y. Lu, S. Y. Liang, J. Zhang, and N. Chi, "Performance analysis of space multiplexing by superposed signal in multi-dimensional VLC system," Optics Express, vol. 26, no. 16, pp. 19762-19772, 2018.

[21] M. Karbalayghareh, F. Miramirkhani, H. B. Eldeeb, R. C. Kizilirmak, S. M. Sait, and M. Uysal, "Channel modelling and performance limits of vehicular visible light communication systems," IEEE Transactions on Vehicular Technology, vol. 69, no. 7, pp. 6891-6901, 2020.
[22] B. Yu, S. Liang, X. Ding, Z. Li, and Y. Tang, "A sandwich structure light-trapping fluorescence antenna with large field of view for visible light communication," IEEE Transactions on Electron Devices, vol. 68, no. 2, pp. 565-571, 2021.

[23] M. Seminara, T. Nawaz, S. Caputo, L. Mucchi, and J. Catani, "Characterization of field of view in visible light communication systems for intelligent transportation systems," IEEE Photonics Journal, vol. 12, no. 4, pp. 1-16, 2020.

[24] N. B. Hassan, Z. Ghassemlooy, S. Zvanovec et al., "Interference cancellation in MIMO NLOS optical-camera-communicationbased intelligent transport systems," Applied Optics, vol. 58, no. 34, pp. 9384-9391, 2019.

[25] P. Luo, Z. Ghassemlooy, H. L. Minh, E. Bentley, A. Burton, and $\mathrm{X}$. Tang, "Performance analysis of a car-to-car visible light communication system," Applied Optics, vol. 54, no. 7, p. 1696, 2015.

[26] S. Gong, X. Lu, D. T. Hoang et al., "Toward smart wireless communications via intelligent reflecting surfaces: a contemporary survey," IEEE Communications Surveys \& Tutorials, vol. 22, no. 4, pp. 2283-2314, 2020.

[27] C. Pan, H. Ren, K. Wang et al., "Intelligent reflecting surface aided MIMO broadcasting for simultaneous wireless information and power transfer," IEEE Journal on Selected Areas in Communications, vol. 38, no. 8, pp. 1719-1734, 2020.

[28] H. Du, J. Zhang, J. Cheng, and B. Ai, "Millimeter wave communications with reconfigurable intelligent surfaces: performance analysis and optimization," IEEE Transactions on Communications, vol. 69, no. 4, pp. 2752-2768, 2021.

[29] S. N. Sur and R. Bera, "Intelligent reflecting surface assisted MIMO communication system: a review," Physical Communication, vol. 47, p. 101386, 2021.

[30] B. B. Dingel, K. Tsukamoto, S. Mikroulis, P. Deng, M. Kavehrad, and Y. Lou, "MEMS-based beam-steerable free-space optical communication link for reconfigurable wireless data center," in Photonics West OPTO Conference on Broadband Access Communication Technologies XI, San Francisco, 2017.

[31] Z. Cao, X. Zhang, G. Osnabrugge, J. Li, I. M. Vellekoop, and A. M. J. Koonen, "Reconfigurable beam system for non-line-of-sight free-space optical communication," Light, science \& applications, vol. 8 , no. 1 , p. 69, 2019.

[32] M. Najafi and R. Schober, "Intelligent reflecting surfaces for free space optical communications," in IEEE Global Communications Conference, Waikoloa, HI, USA, 2019.

[33] A. M. Abdelhady, A. K. S. Salem, O. Amin, B. Shihada, and M.S. Alouini, "Visible light communications via intelligent reflecting surfaces: metasurfaces vs mirror arrays," IEEE Open Journal of the Communications Society, vol. 2, pp. 1-20, 2021.

[34] P. Dutre, P. Bekaert, and K. Bala, Advanced Global Illumination, AK Peters/CRC Press, Boca Raton, FL, USA, 2006.

[35] M. Akanegawa, Y. Tanaka, and M. Nakagawa, "Basic study on traffic information system using LED traffic lights," IEEE Transactions on Intelligent Transportation Systems, Article, vol. 2, no. 4, pp. 197-203, 2001.

[36] T. Komine and M. Nakagawa, "Fundamental analysis for visible-light communication system using LED lights," IEEE Transactions on Consumer Electronics, vol. 50, no. 1, pp. 100-107, 2004. 